\title{
Photoluminescence of InNAs alloys: S-shaped temperature dependence and conduction-band nonparabolicity
}

\author{
M. Merrick, S. A. Cripps, B. N. Murdin, and T. J. C. Hosea* \\ Department of Physics, University of Surrey, Guildford, Surrey GU2 7XH, United Kingdom \\ T. D. Veal and C. F. McConville \\ Department of Physics, University of Warwick, Coventry CV4 7AL, United Kingdom \\ M. Hopkinson \\ Department of Electronic and Electrical Engineering, University of Sheffield, Mappin Street, Sheffield S1 3JD, United Kingdom
}

(Received 14 May 2007; published 29 August 2007)

\begin{abstract}
Photoluminescence (PL) has been used as a means of unambiguously observing band gap reduction in InNAs epilayers grown by molecular beam epitaxy. The observed redshift in room temperature emission as a function of nitrogen concentration is in agreement with the predictions of the band anticrossing (BAC) model, as implemented with model parameters derived from tight-binding calculations. The temperature dependence of the emission from certain samples exhibits a signature non-Varshni-like behavior indicative of electron trapping in nitrogen-related localized states below the conduction-band edge, as predicted by the linear combination of isolated nitrogen states (LCINS) model. This non-Varshni-like behavior tends to grow more pronounced with increasing nitrogen content, but for the highest nitrogen concentration studied, the more familiar Varshni-like behavior is recovered. Although unexpected, this observation is found to be consistent with the BAC and LCINS models. With consideration given to the effects of conduction-band nonparabolicity on the emission line shapes, the BAC model parameters extracted from the measured PL transition energies are found to be in excellent agreement with the predictions of the aforementioned tight-binding calculations.
\end{abstract}

DOI: 10.1103/PhysRevB.76.075209

PACS number(s): 78.55.Cr, 78.66.Fd, 71.55.Eq

\section{INTRODUCTION}

In III-V-based dilute nitride materials, the high electronegativity of the nitrogen atoms substituting for the larger anion in the host matrix (e.g., arsenic, antimony) leads to the formation of a highly localized band of states above the conduction-band minimum (CBM). For dilute nitrogen concentrations, an interaction between these localized states and the extended states of the conduction band leads to a reduction of the fundamental band gap. This interaction is described both by the phenomenological approach of the band anticrossing (BAC) model ${ }^{1}$ and by complementary detailed band structure calculations (see, for instance, Ref. 2). These models have been successfully used both to explain and to predict the observed band structure of several dilute nitride alloys such as GaNAs, GaInNAs, and InNP. ${ }^{3}$ Generally, efforts have tended to focus on GaInNAs due to its potential use in commercially important near-infrared applications such as telecommunications. Although interest in material systems capable of emitting and/or detecting at midinfrared wavelengths has grown steadily in recent years, due largely to the relevance of such wavelengths to molecular gas sensing applications, the study of suitable narrow gap semiconductor-based dilute nitride alloys, such as InNAs and GaNSb, remains in its infancy. ${ }^{4,5}$

Given the well-known proclivity of the InAs host matrix toward degenerate $n$-type doping, it is reasonable to expect that band-filling effects will play a role in InNAs. ${ }^{4}$ Indeed, the $n$-type conductivity of InNAs has been demonstrated previously by Hall effect measurements of epilayer material grown on semi-insulating GaAs substrates. ${ }^{6,7}$ In the presence of degenerate electron concentrations, optical characterization techniques that probe the absorption edge are generally incapable of resolving transitions between band edge states. Generally, the Moss-Burstein shift (band filling) tends to dominate measured spectra, leading to a blueshift of the absorption edge with respect to the band gap. Such effects have, indeed, proven extremely problematic in previous attempts to measure band gap reduction directly in InNAs using absorption spectroscopy, with large blueshifts being observed rather than any redshift. ${ }^{6-9}$ The Moss-Burstein shifts were "subtracted" during analysis to infer a net redshift in band gap. ${ }^{7-9}$ An unambiguous redshift of the absorption edge of InNAs alloys with increasing nitrogen content has been seen only in one previous study. ${ }^{10}$

In a previous preliminary study, we used photoluminescence (PL) spectroscopy to investigate the nitrogen-induced band gap reduction in InNAs samples containing nitrogen percentages $(N)$ of $1.0 \%$ and $2.2 \%$ at temperatures between 77 and $300 \mathrm{~K} .{ }^{4}$ Rather than probing the absorption edge, PL spectroscopy measures the light emitted by a recombining population of thermalized carriers. Band-to-band PL emission, thus, originates predominantly from transitions between states close to the band edges, making PL techniques less sensitive to the effects of degenerate doping. We were, therefore, able to observe an unambiguous nitrogen-induced band gap reduction in the two InNAs alloys. ${ }^{4}$ Here, PL and PLlineshape analyses are employed to probe the optical properties of a more comprehensive set of InNAs epilayers over an extended temperature range of $15-300 \mathrm{~K}$. 


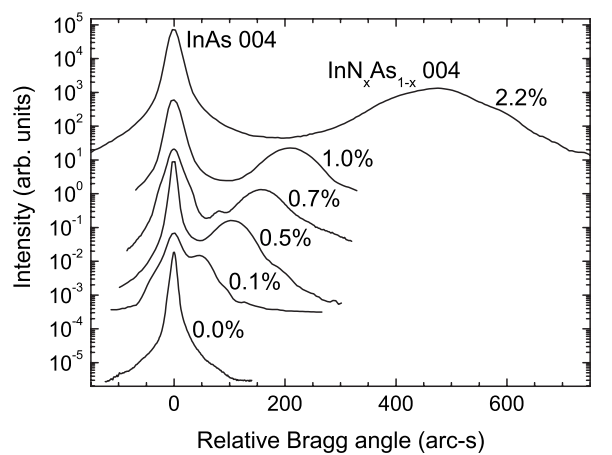

FIG. 1. X-ray diffraction of the 004 diffraction maximum from both the 300-nm-thick InNAs epilayers and the InAs substrates. The curves have been offset vertically on the logarithmic scale by multiplication. Note that the $N=0.4 \%$ sample is omitted for clarity.

\section{EXPERIMENTAL DETAILS}

The $\mathrm{InN}_{x} \mathrm{As}_{1-x}$ epilayers studied were grown pseudomorphically to a thickness of $300 \mathrm{~nm}$ on Zn-doped p-type InAs(001) substrates by molecular beam epitaxy (MBE) using a turbo-pumped Vacuum Generators V80 system equipped with an Oxford Applied Research HD25 rf plasma nitrogen source. The substrate temperature was within the range $350-400{ }^{\circ} \mathrm{C}$ for the growth of all the InNAs samples. A 300-nm-thick InAs epilayer was also grown using a substrate temperature of $460{ }^{\circ} \mathrm{C}$. The amount of nitrogen incorporated in the different films was varied by tuning the plasma power and nitrogen gas pressure. The nitrogen content in the InNAs layers was determined by high-resolution x-ray diffraction (XRD) of the symmetric 004 reflection. The XRD curves from the InNAs layers are shown in Fig. 1. The separation between the substrate and epilayer diffraction peaks indicates the change in lattice constant in the epilayer due to nitrogen incorporation, which, via Vegard's law, gives the nitrogen content, as indicated in Fig. 1.

Spectral measurements of the PL emission from each sample were made at several temperatures between 15 and $300 \mathrm{~K}$. The samples were mounted on the cold finger of a Cryophysics closed-cycle helium cryostat. Although the quartz glass cryostat window is not ideal for midinfrared applications, steps were taken to ensure its long wavelength cutoff did not adversely affect the measured spectra. Corrections were made for both window response and atmospheric absorption during data analysis. A mechanically chopped, diode-pumped neodymium: yttrium-aluminum-garnet laser, outputting $\sim 100 \mathrm{~mW}$ at a wavelength of $1064 \mathrm{~nm}$, was used as the excitation source. Hence, states well above the conduction-band edge are populated in the studied materials. The PL emission was spectrally resolved using a Bomem fast-scanning Fourier transform infrared spectrometer and detected by a liquid-nitrogen-cooled InSb detector coupled to a lock-in amplifier. Since the maximum fringe modulation frequency of the Bomem (arising from the mirror scanning motion) was approximately $3.3 \mathrm{kHz}$, it was necessary to chop the laser at a relatively high frequency of $\sim 20 \mathrm{kHz}$. The diameter of the laser spot at the sample position was approximately $1 \mathrm{~mm}$, which corresponds to a power density of $\sim 10 \mathrm{~W} \mathrm{~cm}^{-2}$.

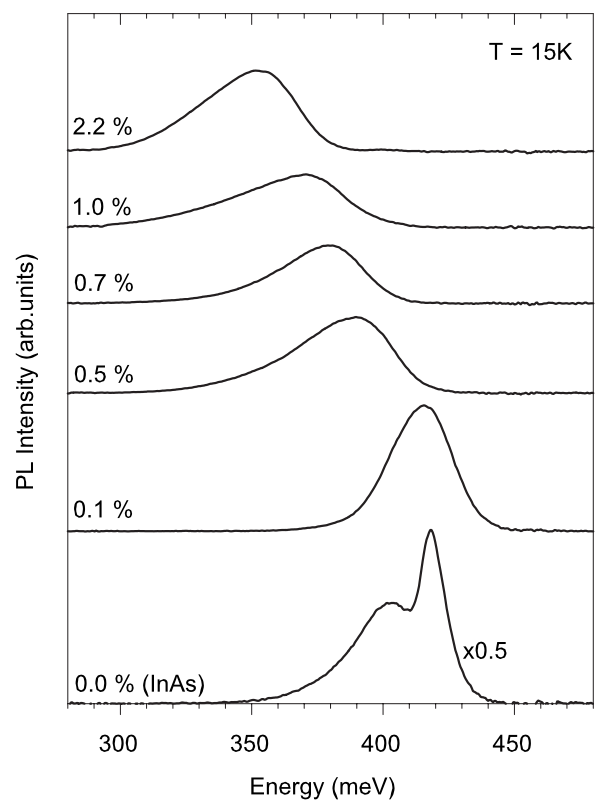

FIG. 2. Low temperature (15 K) PL emission from all the epilayer samples (except that the $N=0.4 \%$ sample is omitted for clarity). Apart from the $0 \%$ sample, the spectra are not normalized or scaled, though their base lines are offset for clarity. The doublepeaked line shape of the InAs $(N=0.0 \%)$ spectrum is consistent with the results of another PL study of MBE-grown InAs in the literature (Ref. 25), in which the higher energy peak ( $418 \mathrm{meV}$ here) is attributed to the actual band-to-band recombination. A consistent, unambiguous shift of the emission toward lower energy is observed with increasing $N$.

\section{RESULTS}

It can clearly be seen in Fig. 2 that the low temperature PL emission shifts steadily and unambiguously to lower energy as the epilayer nitrogen content is increased. As shown in the first two frames of Fig. 3, for those samples with moderate $N$ (i.e., $0.5 \%$ and $1.0 \%$ ), a non-Varshni-like evolution of the PL peak energy is observed with increasing temperature; i.e., on heating from low temperature, the PL peak initially shifts toward higher energies before slowing and eventually reversing its direction toward lower energies. This so-called S-shaped temperature dependence is a signature behavior of dilute nitride materials and has been observed in several such semiconductor alloys. ${ }^{11,12}$

To understand the origin of the S-shaped temperature dependence, one must consider the local environment of the substitutional nitrogen atoms in the lattice. For low $N$, and to a first approximation, interactions between the sparsely populated substitutional nitrogen atoms are negligible (i.e., there is no significant overlap of their electronic wave functions). Within the framework of the BAC model, the interaction between the localized band of isolated nitrogen states, whose energies $E_{N}^{0}$ are measured relative to the valence band maximum (VBM), and the extended band of unperturbed host conduction states, with energies $E_{M}^{0}(\mathbf{k})$ also relative to the VBM, may then be described by the following $2 \times 2$ matrix: 


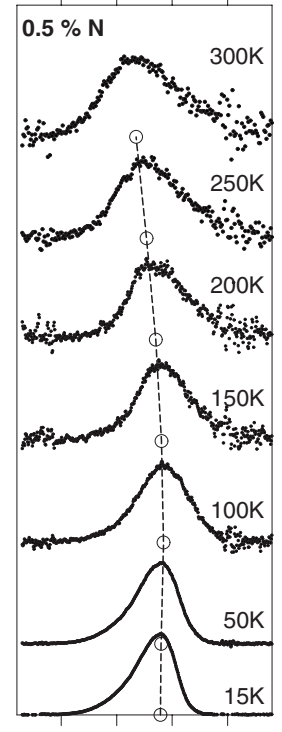

300350400450

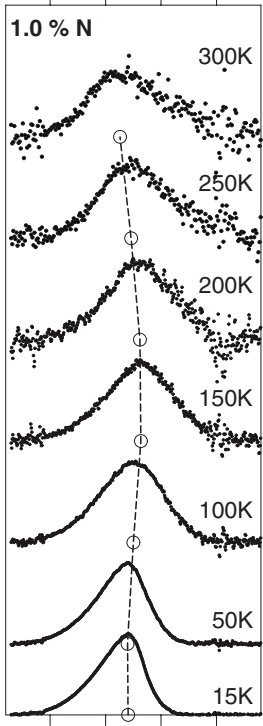

300350400450

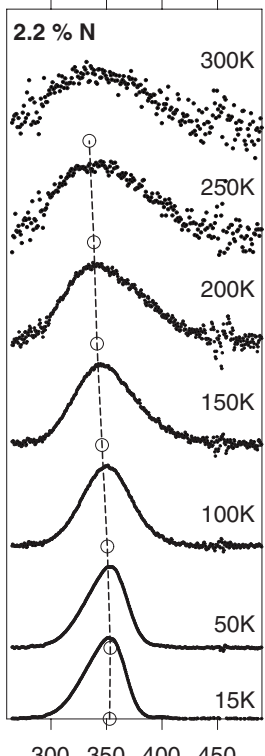

$300 \quad 350 \quad 400 \quad 450$

\section{Energy (meV)}

FIG. 3. Temperature dependent PL emission from a selection of samples with $0.5 \% \leqslant N \leqslant 2.2 \%$. All peak heights have been normalized to be equal, and offset vertically for clarity. The open circles indicate the peak positions and lie at the base lines of the spectra to which they relate. The "S-shaped" dependence of the PL peak energies (vertical dashed curves), observed only weakly for $N=0.5 \%$ (left), is significantly more pronounced for $N=1.0 \%$ (middle), consistent with an increase in the density of BAC states with added nitrogen. However, at $N=2.2 \%$ (right), this effect is no longer apparent and a more familiar Varshni-like evolution of the PL peak energy with temperature is recovered.

$$
\left(\begin{array}{cc}
E-E_{M}(\mathbf{k}) & -\beta x^{1 / 2} \\
-\beta x^{1 / 2} & E-E_{N}^{0}
\end{array}\right),
$$

where $x$ is the molar fraction of nitrogen present in the alloy, $\beta$ parametrizes the energy of the BAC interaction, and $E_{M}(\mathbf{k})$ are the energies of the perturbed conduction-band states given by $E_{M}(\mathbf{k})=E_{M}^{0}(\mathbf{k})-\alpha x$, where $\alpha$ parametrizes the perturbation experienced by the extended states due to the presence of nitrogen "impurities" in the lattice. The eigenvalues $E$ can then be found by equating the determinant of the above matrix to zero, giving the following quadratic solutions:

$$
E_{ \pm}(\mathbf{k})=\frac{\left[E_{N}^{0}+E_{M}(\mathbf{k})\right] \pm\left\{\left[E_{N}^{0}-E_{M}(\mathbf{k})\right]^{2}+4 x \beta^{2}\right\}^{1 / 2}}{2},
$$

where the two conduction subbands $E_{-}(\mathbf{k})$ and $E_{+}(\mathbf{k})$ are such that $E_{-}(\mathbf{k}=0)<E_{M}(\mathbf{k}=0)$ and $E_{+}(\mathbf{k}=0)>E_{N}^{0}$. Then, because $E_{-}(\mathbf{k}=0)$ represents the new effective CBM, the (redshifted) band gap is redefined as the energy gap between the VBM and $E_{-}(\mathbf{k}=0)$.

As more nitrogen atoms are added to the lattice there is an increased probability of two or more being bonded to the same indium atom, so that there is significant overlap of their electronic wave functions, leading to the formation of nitro- gen "complexes." There are a large number of unique complex configurations available in the ternary tetrahedral lattice of a III-N-V semiconductor, each of which has a different self-energy lower than $E_{N}^{0}$ [and crucially, therefore, closer to being in resonance with $\left.E_{M}(\mathbf{k}=0)\right]$. BAC model-type interactions between these nitrogen complexes and $E_{M}(\mathbf{k})$, thus, form an array of localized states below $E_{-}(\mathbf{k}=0)$, which we shall refer to as "BAC states." A quantitative analysis of the formation of such BAC states in GaNAs has been published by Lindsay and O'Reilly, where an approach referred to as the linear combination of isolated nitrogen states (LCINS) model was used. ${ }^{13}$ In that work, it was suggested that, for $N<5.0 \%$, there is a linear evolution of the average nitrogen state energy $E_{N}$ as $N$ is increased, so that $E_{N}^{0}$ in Eq. (2) can be replaced by $E_{N}=E_{N}^{0}-\gamma x$, where $\gamma$ is a positive constant. Although a similarly thorough quantitative analysis does not yet exist for InNAs, one is encouraged by the structural similarities between the GaAs and InAs host lattices to assume a similar evolution of $E_{N}$ as a function of $N$, so that $E_{N}^{0}$ in Eq. (2) can also be replaced by $E_{N}=E_{N}^{0}-\gamma x$.

Consider now the temperature dependence of the PL emission spectrum that might result from the band structure described above on heating from, say, absolute zero: at very low temperatures, it is expected that there should be a significant below-band-gap contribution from the recombination of electrons trapped in the BAC states. On heating, however, thermal excitation of these trapped electrons into the conduction band should gradually reduce the below-band-gap recombination, thereby shifting the PL emission peak to higher energy, closer to the actual band gap energy. This behavior, thus, contributes a "non-Varshni" component to the temperature dependence of the peak emission energy. The normal Varshni-like decrease of the (III-V) semiconductor band gap with temperature ${ }^{14}$ will compete with this non-Varshni component as temperature is raised, but at low temperatures, the weak redshift due to the former will be outweighed by the blueshift due to the latter. However, while the rate of the redshift increases progressively with further heating, that of the blueshift will decrease due to the exponential drop in the occupancy of the BAC states. Hence, in InNAs, the rate of the initial blueshift of the PL emission peak energy with increasing temperature should first slow down before halting and eventually giving way to a redshift.

The preceding discussion, thus, provides a qualitative understanding of the mechanism responsible for the S-shaped variation (or bowing) of the PL peak energy as a function of temperature exhibited by the $N=0.5 \%$ and $N=1.0 \%$ samples (as shown in the first two panels of Fig. 3). From Fig. 3, we also note that increasing $N$ from $0.5 \%$ to $1.0 \%$ enhances the $\mathrm{S}$-shaped bowing. This observation is also consistent with the above qualitative argument, since adding nitrogen will increase the number of nitrogen complexes, which in turn will increase the density of the BAC states, and, hence, the amplitude of the non-Varshni component of the temperature dependence. However, Fig. 3 also shows that the highest nitrogen content epilayer studied, $N=2.2 \%$, displays little or no S-shaped behavior. To understand this result, consider the evolution of the average energy of the conduction-band states $E_{-}(\mathbf{k})$, as defined by Eq. (2), relative to the energy of the BAC states. As nitrogen is added to the alloy, $E_{-}(\mathbf{k}=0)$ 
decreases while the energies of pair states, by far the most probable order of complex, remain approximately constant relative to the VBM. When $E_{-}(\mathbf{k}=0)$ comes within $\sim k_{B} T$ of the energy of a given pair state, the two effectively become amalgamated. That is to say, as $N$ is increased through this transitional range (which one can infer from our results occurs here between $1.0 \%$ and $2.2 \%$ ), the distinction between the extended $E_{-}(\mathbf{k})$ states and pair states becomes progressively blurred until, ultimately, the two are indistinguishable. The amplitude of the non-Varshni component would, thus, diminish accordingly, reducing the amplitude of the S-shaped bowing. For $N>2.2 \%$, only states arising from significantly less probable higher order complexes (e.g., triplet states) are distinguishable as BAC states, which will be encountered as $E_{-}(\mathbf{k}=0)$ continues to decrease with added nitrogen. The waxing and waning of the S-shaped bowing seen in Fig. 3 is, therefore, expected to repeat as $N$ is further increased.

\section{ANALYSIS AND DISCUSSION}

In order to analyze our PL spectra to obtain, in particular, the band gap energy $E_{g}$ as a function of $N$, we shall next restrict the description of our analysis of the present measurements to the $300 \mathrm{~K}$ PL spectra only. The reasons for this will be explained later.

There are a number of procedures in the literature for obtaining the band gap transition energy from a band-to-band PL feature. The majority of these simplify the analysis by assuming either a parabolic dispersion for both the conduction and valence bands, or, even more simply, that the PL peak position is essentially equal to the transition energy. ${ }^{15-18}$ Such simplifications ignore the effects of band nonparabolicity, despite the intrinsically nonparabolic nature of the conduction band in InAs being well documented. ${ }^{19-21}$ Indeed, provided the experimental probe is insensitive to states far from the CBM, as is usually assumed in PL spectroscopy, such simplifications are often quite reasonable: it is a nontrivial task to include the corrections necessary to take account of intrinsic nonparabolicity. However, InNAs is expected to have an additional contribution to non-parabolicity, over and above that of InAs, due to the BAC interaction. Additionally, its high $n$-type doping is likely to result in a significant occupation of the higher-lying conduction-band states near room temperature, where the effects of a nonparabolic dispersion are enhanced. Hence, we consider it important to evaluate the influence of nonparabolicity on the line shape of the band-to-band PL emission in this material system in order to give increased confidence in the transition energies we shall obtain here.

To that end, we next describe the development of a methodology that combines a least-squares fitting procedure using an analytical parabolic model for the PL line shape function, with a nonparabolic correction derived from a calculated "predicted" PL line shape model. We will show that, although both PL line shape models have essentially the same basic form, the introduction of nonparabolicity means that one cannot write down suitable analytical expressions in this case, and one must resort to numerical methods to complete the analysis. As a starting point, we calculate the nonpara-

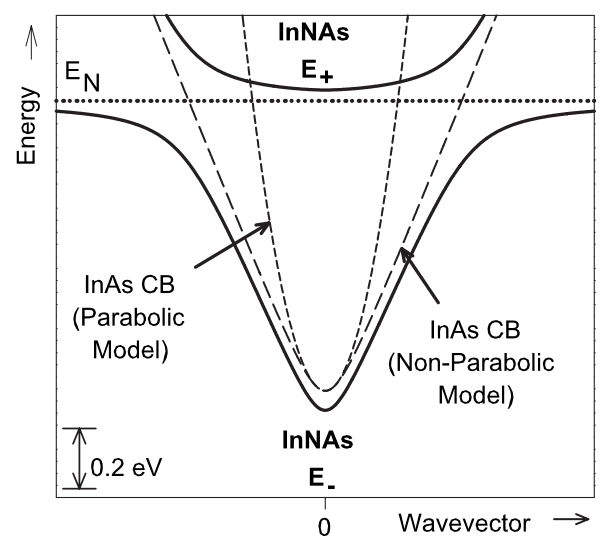

FIG. 4. E-k plot of the calculated nonparabolic conduction band (CB) dispersions of InAs (long-dashed curve) and InNAs (solid curves) for $N=1.0 \%$. The parabolic approximation for the InAs conduction band [using the accepted band edge effective mass (Ref. 23)] is also shown for comparison (short-dashed curve). For states $>\sim 0.1 \mathrm{eV}$ above the InAs CBM, parabolicity is clearly a poor approximation to the more realistic nonparabolic dispersion. The dispersion for $N=1.0 \%$ InNAs has been calculated by substituting Eq. (3) into Eq. (2). As may be seen, the BAC interaction enhances the nonparabolicity only slightly over that of the binary InAs. However, when coupled with the proclivity of InNAs for high $n$-type degenerate doping, which can lead to the occupation of states well above the CBM, there is significant potential for those states with nonparabolic dispersion to contribute to band-to-band PL emission.

bolic conduction-band dispersion of the host InAs using the following eigenvalue of the Pidgeon-Brown Hamiltonian matrix: ${ }^{22}$

$$
E_{M}\left(E_{k 0}\right)=E_{g}^{\mathrm{InAs}}+E_{k 0}\left\{\frac{E_{g}^{\mathrm{InAs}}}{2 E_{k 0}}\left[\sqrt{1+\frac{8 E_{p} E_{k 0}}{3\left(E_{g}^{\operatorname{InAs}}\right)^{2}}}-1\right]+1\right\},
$$

where $E_{g}^{\mathrm{InAs}}$ is the InAs band gap energy, $E_{p}$ parametrizes the strength of the $\mathbf{k} \cdot \mathbf{p}$ mixing interaction between conduction and valence band states [where $E_{p}=21.5 \mathrm{eV}$ for InAs (Ref. 23)], and $E_{k 0}=(\hbar k)^{2} / 2 m_{0}$ is the kinetic energy of a free electron. The InNAs conduction-band dispersion may then be calculated by substituting this nonparabolic form for $E_{M}^{0}$ into Eq. (2) [recalling that $E_{M}(\mathbf{k})=E_{M}^{0}(\mathbf{k})-\alpha x$ ]. Figure 4 shows an example of these calculations for InAs and InNAs with $N=1.0 \%$. This clearly illustrates the deviations from parabolicity at energies greater than $\sim 100 \mathrm{meV}$ above the respective CBMs.

The PL emission from a semiconducting alloy at a given photon energy $\hbar \omega$ is proportional to the joint density of states $\rho$ linking the initial $\left(E_{1}\right)$ and final $\left(E_{2}\right)$ states, which can be calculated using the following well-known general expression: ${ }^{22}$

$$
\rho_{\left(\hbar \omega=E_{2}-E_{1}\right)}=\frac{1}{2 \pi^{2}}\left(\frac{2 m_{0}}{\hbar^{2}}\right)^{3 / 2}\left(\frac{d E_{1}}{d E_{k 0}}+\frac{d E_{2}}{d E_{k 0}}\right)^{-1} E_{k 0}^{1 / 2},
$$

where, for band-to-band recombination in InNAs, $E_{1}$ is in the $E_{-}(\mathbf{k})$ band and $E_{2}$ is assumed to be in the heavy hole 
valence band. We further assume that the heavy hole valence band is parabolic, with a negative effective mass of $-0.41 m_{0},{ }^{23}$ giving a constant value for $d E_{2} / d E_{k 0}$ of $(-0.41)^{-1}$. Differentiating the negative solution of Eq. (2) with respect to $E_{k 0}$ yields the following analytical expression for $d E_{1} / d E_{k 0}$ in InNAs

$$
\begin{aligned}
\frac{d E_{1}}{d E_{k 0}}= & \frac{1}{2} \frac{d E_{M}}{d E_{k 0}}\left(1+\left\{\left[E_{N}-E_{M}\left(E_{k 0}\right)\right]^{2}+4\left(\beta x^{1 / 2}\right)^{2}\right\}^{-1 / 2}\right. \\
& \left.\times\left[E_{N}-E_{M}\left(E_{k 0}\right)\right]\right),
\end{aligned}
$$

where the required $d E_{M} / d E_{k 0}$ may be obtained from Eq. (3). Finally, Eq. (5) may then be substituted into Eq. (4) to yield the required nonparabolic (NP) joint density of states $\rho_{N P}$.

Here, we perform least-squares fits to the $300 \mathrm{~K}$ PL spectra measured for each epilayer, using a functional form $I_{P L}(\hbar \omega)$, described next. Due to the unintentional degenerate doping of the InNAs material, we assume that Fermi-Dirac functions $f_{e}$ and $f_{h}$ are appropriate for the electron and hole occupations, respectively (applying Boltzmann statistics in such circumstances would lead to an overestimation of the band-to-band energy gap $E_{g}$ ). We use the following functional form for our PL line shape model, which incorporates an Urbach tail for below-band-edge emission: ${ }^{24}$

$$
\begin{gathered}
I_{P L}\left(\hbar \omega \leqslant E_{c r}\right)=A K \exp \left[\frac{\sigma}{k_{B} T}\left(\hbar \omega-E_{c r}\right)\right] f_{e} f_{h}, \\
I_{P L}\left(\hbar \omega>E_{c r}\right)=A \rho f_{e} f_{h},
\end{gathered}
$$

where $E_{c r}$ is the energy of the transition (or crossover) between Eqs. (6a) and (6b), $K$ a dummy parameter chosen to ensure that the transition is a smooth one, $T$ the carrier temperature, $\sigma$ a dimensionless phenomenological parameter describing the slope of the Urbach tail, and $A$ is related to the Einstein coefficient linking the conduction and valence band states (but is treated here as a fitting parameter).

Before considering the effects of using a fully nonparabolic model for the PL line shape, let us examine what happens when we make the simplest choice for $\rho$ in Eq. (6b), i.e., parabolic conduction and valence band dispersions. Then, $d E_{1} / d E_{k 0}$ and $d E_{2} / d E_{k 0}$ in Eq. (4) are both constants, so that $\rho$ is directly proportional to $E_{k 0}^{1 / 2}$ and the familiar parabolic joint density of states $\rho_{\text {para }} \propto\left(\hbar \omega-E_{g}\right)^{1 / 2}$ is recovered. To obtain the required smooth transition between Eqs. (6a) and (6b), it is necessary that both their amplitudes and first derivatives with respect to photon energy be continuous at $\hbar \omega=E_{c r}$. For $\rho=\rho_{\text {para }}$, it is straightforward to show that these continuity conditions require that $E_{c r}=E_{g}+k_{B} T / 2 \sigma$ and $K=\left(k_{B} T / 2 \sigma\right)^{1 / 2}$. Hence, $I_{P L}(\hbar \omega)$ takes on a simple analytical form that can easily be programed into a computer for performing least-squares fits to experimental spectra. Figure 5 shows an example of fitting this parabolic-dispersion-based model to the measured PL spectrum for the $N=1.0 \%$ sample at $300 \mathrm{~K} ; A, E_{g}, T$, and $\sigma$ were all allowed to vary as fit parameters, as were $E_{e}$ and $E_{h}$, the quasi-Fermi-energies in $f_{e}$ and $f_{h}$ (defined relative to the respective band edges). Similar satisfactory fits to the $300 \mathrm{~K}$ PL spectra of all the samples were obtained using this parabolic model. However, as pre-

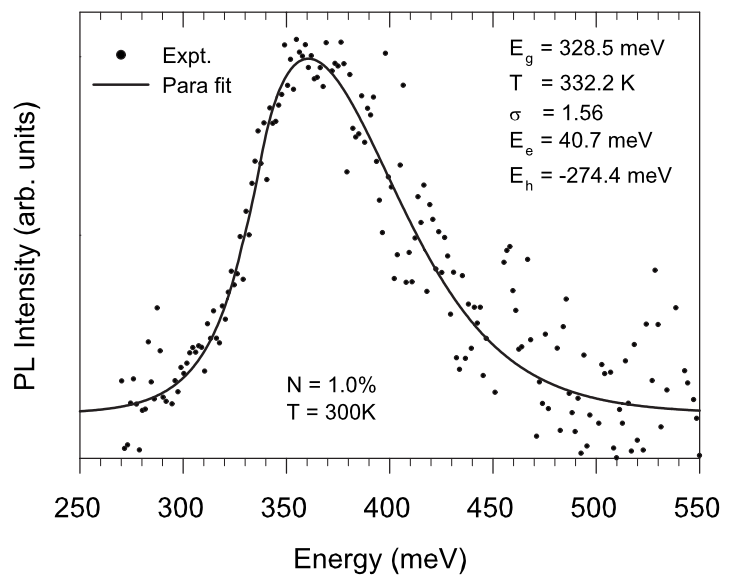

FIG. 5. The full curve shows a fit of the parabolic PL lineshape model [Eqs. (6a) and (6b) with $\rho=\rho_{\text {para }}$ ] to the measured PL emission (dots) from the $N=1.0 \%$ epilayer at $300 \mathrm{~K}$. Here, $A, E_{g}, T, \sigma$, and the quasi-Fermi-energies $E_{e}$ and $E_{h}$ (measured relative to the $\mathrm{CBM}$ and VBM, respectively) were allowed to vary as fit parameters, with the resulting values shown on the upper right corner of the figure.

viously remarked, these results must be treated with due caution since the effects of a nonparabolic conduction-band dispersion (e.g., as in Fig. 4) on the line shapes of the InNAs PL spectra have been ignored.

Consequently, we now turn to the physically more realistic situation incorporating a nonparabolic conduction-band dispersion. Unfortunately, it is found that one cannot simply apply the above procedure when using a nonparabolic joint density of states $\rho_{N P}$ : if $d E_{1} / d E_{k 0}$ in Eq. (4) is defined by Eq. (5) [with Eq. (3) being needed to obtain $d E_{M} / d E_{k 0}$ ], it is then not possible analytically to solve the continuity requirements of Eqs. (6a) and (6b) to obtain expressions for $K$ and $E_{c r}$. As a result, one cannot write down a simple computer programmable function with which to perform the least-squares fitting. However, there is a possible route out of this apparent dilemma. For a given set of parameters $\sigma, T, E_{g}, E_{e}$, and $E_{h}$, together with the nonparabolic density of states function $\rho_{\mathrm{NP}}$, it is possible to use iterative numerical techniques to find values for $E_{c r}$ and $K$ that satisfy the continuity requirements of Eqs. (6a) and (6b). Hence, by using the best available predictions for the InNAs BAC model parameters, and fully incorporating conduction-band nonparabolicity, one can generate a predicted PL line shape representative of any given alloy composition. By comparing this with the corresponding measured PL spectrum, one may then manually adjust the phenomenological parameters $\sigma$ (which describes only the slope of the below-band-gap Urbach tail) and $A$ (effectively an intensity scaling factor) to try to obtain a visually reasonable match. Although this may give a reasonably good approximation to the measured PL spectrum, it is by no means a "fit." However, we can next perform a least-squares fit to this predicted nonparabolic model PL line shape using the aforementioned parabolic model PL line shape. Then, by comparing the resulting parabolic fit parameters with the original values used to generate the nonparabolic line shape, one may obtain a quantitative estimate of the errors in the 
TABLE I. Comparison of the original nonparabolic (NP) model parameters used to generate nonparabolic $300 \mathrm{~K}$ InNAs PL line shapes for $N=0.5 \%, 1.0 \%$, and $2.2 \%$ to obtain an approximate representation of the experimental spectra (see, e.g., main plot in Fig. 6 for $N=1.0 \%$ ), and the result of fitting those generated line shapes with an analytical line shape model that assumes a parabolic conduction-band dispersion ("para fit," see, e.g., the inset of Fig. 6). Clearly, the fit parameters deviate increasingly from their original NP values as $N$ is increased. In particular, $E_{g}$ is overestimated by $\sim 1.1 \mathrm{meV}$ for $N=0.5 \%, \sim 2.0 \mathrm{meV}$ for $N=1.0 \%$, and $\sim 5.7 \mathrm{meV}$ for $N=2.2 \%$. It is shown in Fig. 7 that this "error" in $E_{g}\left(\Delta E_{g}\right)$ increases roughly as the square of $N$.

\begin{tabular}{|c|c|c|c|c|c|c|}
\hline \multirow[b]{2}{*}{ Parameter } & \multicolumn{2}{|c|}{$\begin{array}{c}N=0.5 \% \\
\left(n=2 \times 10^{17} \mathrm{~cm}^{-3}\right)^{\mathrm{a}}\end{array}$} & \multicolumn{2}{|c|}{$\begin{array}{c}N=1.0 \% \\
\left(n=4 \times 10^{17} \mathrm{~cm}^{-3}\right)^{\mathrm{a}}\end{array}$} & \multicolumn{2}{|c|}{$\begin{array}{c}N=2.2 \% \\
\left(n=1 \times 10^{18} \mathrm{~cm}^{-3}\right)^{\mathrm{a}}\end{array}$} \\
\hline & NP & Para fit & NP & Para fit & NP & Para fit \\
\hline$E_{g}(\mathrm{meV})$ & 336 & 337 & 322 & 324 & 289 & 295 \\
\hline$T(\mathrm{~K})$ & 300 & 326 & 300 & 319 & 300 & 308 \\
\hline$\sigma$ & 1.1 & 1.5 & 1.0 & 1.3 & 0.8 & 0.9 \\
\hline$E_{e}(\mathrm{meV})$ & +10 & +16 & +34 & +41 & +75 & +80 \\
\hline$E_{h}(\mathrm{meV})$ & -346 & -338 & -357 & -299 & -364 & -345 \\
\hline
\end{tabular}

aalues used for $n$-type doping concentration based on data from Ref. 9 for InNAs alloys with similar compositions.

parabolic fit parameters, relative to the original (or "true") nonparabolic values, incurred by least-squares fitting the experimental line shapes with the parabolic model.

In what follows, we demonstrate the implementation of this procedure. The following BAC model parameters have been derived from tight-binding calculations: ${ }^{4} \alpha=1.30 \mathrm{eV}$, $\beta=1.30 \mathrm{eV}, \gamma=2.00 \mathrm{eV}$, and $E_{N}^{0}=1.36 \mathrm{eV}$. For $E_{g}^{\mathrm{InAs}}$ in Eq. (3), a representative value of $350 \mathrm{meV}$ was chosen, which is consistent with suggested literature values. ${ }^{23}$ Theoretical nonparabolic PL line shapes were then generated for $N$ $=0.0 \%$ (i.e., InAs), $0.5 \%, 1.0 \%$, and $2.2 \%$. For these nitrogen concentrations, the $E_{e}$ and $E_{h}$ quasi-Fermi-energies of Eqs. (6a) and (6b) were calculated based on literature data for $n$ (see Table I) in InNAs alloys with similar compositions. ${ }^{9}$ (Parallel conduction through the InAs substrates prevented reliable Hall effect measurements of the actual $n$ in the present InNAs epilayers.) The final phenomenological parameters $A$ and $\sigma$ were then adjusted manually to obtain a visually reasonable match with the corresponding experimental spectra. These predicted (nonparabolic) PL line shapes were then least-squares fitted using the parabolic line shape model.

An example of a nonparabolic line shape generated to match the measured $N=1.0 \%$ experimental spectrum is shown in Fig. 6. Evidently, a satisfactory approximate agreement has been obtained. Note that the BAC model parameters predicted for the given alloy composition will be unlikely to yield a perfect match between the predicted and measured PL peak energies, so one should expect some small relative energy displacement of these two spectra, as is indeed observed in Fig. 6. The inset of Fig. 6 then shows the result of least-squares fitting the parabolic model to this generated nonparabolic PL spectrum. It may be seen that these two line shapes are very closely matched. What is different, however, are the two sets of parameters used to generate the respective spectra. Table I gives a comparison of the original parameters used to generate the nonparabolic model PL spectrum, and their fitted parabolic counterparts for $N=0.5 \%$,
$1.0 \%$, and $2.2 \%$. As may be seen, the fitted parabolic parameters are all different from the original nonparabolic values, with the discrepancies showing a clear increasing trend with increasing $N$. The parameter of greatest interest here is $E_{g}$, which, for $N<0.5 \%$, is perturbed by an amount $\Delta E_{g}$ $\approx+1.1 \mathrm{meV}$ from its true value. This perturbation is compa-

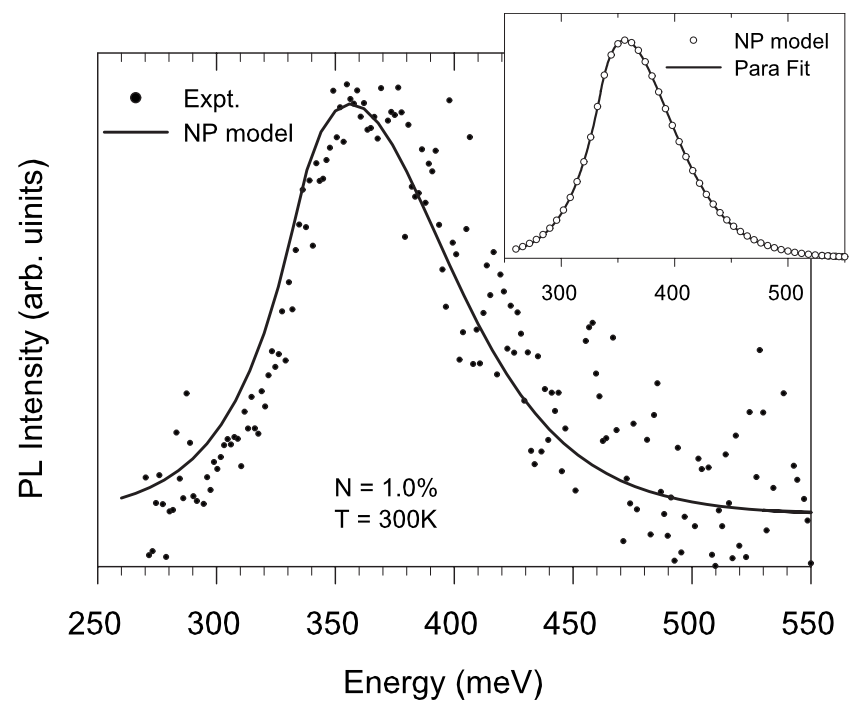

FIG. 6. Main figure: The dots show the measured PL from the $N=1.0 \%$ epilayer at $300 \mathrm{~K}$ (as in Fig. 5), while the solid curve shows a calculated nonparabolic PL line shape generated by substituting $\rho=\rho_{N P}$ into Eqs. (6a) and (6b), and solving numerically for $E_{c r}$ and $K$. The required BAC model parameters were derived from tight-binding calculations, while the other parameters in Eqs. (6a) and (6b) were adjusted for a visually reasonable match with the experimental spectrum. Inset: The same generated nonparabolic line shape (now shown as circles) but least-squares fitted with the analytical parabolic form of the model (solid curve), i.e., by substituting $\rho=\rho_{\text {para }}$ into Eqs. (6a) and (6b). The original NP parameters $E_{g}$, $T, \sigma, E_{e}$, and $E_{h}$, and their fitted parabolic counterparts, are compared in Table I. 


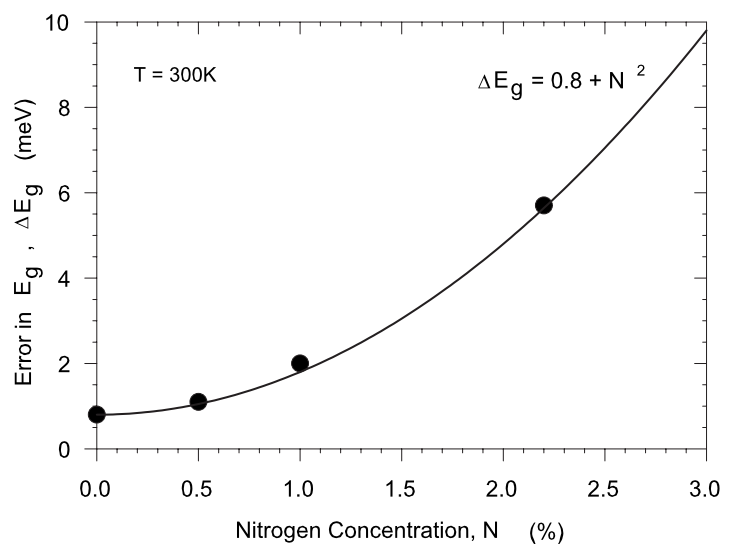

FIG. 7. The estimated error in $E_{g}\left(\Delta E_{g}\right.$, filled circles) as a function of $N$ due to fitting the parabolic-dispersion-based PL line shape model to the experimental band-to-band PL spectra of InNAs (which is expected to be affected by a nonparabolic conductionband dispersion). This error was obtained by fitting the parabolic PL line shape model to a set of calculated nonparabolic PL line shapes (as shown, for example, by the inset in Fig. 6 and example results given in Table I). The error is seen to result in an overestimation of $E_{g}$ compared to the "true" nonparabolic value, which increases roughly as the square of $N$ [i.e., $E_{g}($ true $)=E_{g}($ fit $)-\left(0.8+N^{2}\right) \mathrm{meV}$ ], as shown by the empirical quadratic curve. Although for $N$ $<1.0 \%$ the error is comparable to the statistical error in a parabolic model fitted to the measured PL emission, at $N=2.2 \%$ it is experimentally significant, $\sim 5.7 \mathrm{meV}$.

rable to the statistical uncertainty associated with fitting the parabolic model line shape to the experimental spectra. Hence, the parabolic model could be used to fit such $300 \mathrm{~K}$ PL spectra with some degree of confidence. At $N=2.2 \%$, however, a more statistically significant perturbation of $\Delta E_{g} \approx+5.7 \mathrm{meV}$ is found.

It is unlikely that the nitrogen-induced enhancement in the conduction-band effective mass is responsible for the larger $\Delta E_{g}$ in the $N=2.2 \%$ case: in InNAs, for state energies within $100 \mathrm{meV}$ of the CBM, $d E_{1} / d E_{k 0}$ in Eq. (4) is extremely insensitive to $N$ for $0 \% \leqslant N \leqslant 2.2 \%$. Rather, the dominant factor is the increased occupation of states far above the CBM, which is manifested in our model as a rapidly increasing quasi-Fermi-energy $E_{e}$ as a function of $N$ (see Table I): e.g., for $N=2.2 \%$ and using a literature value ${ }^{9}$ for $n=1$ $\times 10^{18} \mathrm{~cm}^{-3}$, our calculations predict that $E_{e}$ lies $\sim 80 \mathrm{meV}$ above the CBM. Taking into account the electrons' thermal energy at $300 \mathrm{~K}\left(k_{B} T \sim 25 \mathrm{meV}\right)$, this means, therefore, that there may be significant occupation of states up to $\sim 100 \mathrm{meV}$ above the CBM. Inspection of Fig. 4 shows that such states lie within the region where nonparabolicity is predicted to become important. This conclusion, thus, emphasizes the critical role played by band-filling effects in determining the optical properties of InNAs.

In Fig. 7, we plot the "error" $\Delta E_{g}$ due to fitting a nonparabolic PL feature with the parabolic line shape model as a function of $N$. This suggests that $\Delta E_{g}$ increases empirically as the square of $N$. Therefore, the key result of this analysis is that, in order to account for the effects of nonparabolicity on the line shape of band-to-band PL emission from bulk

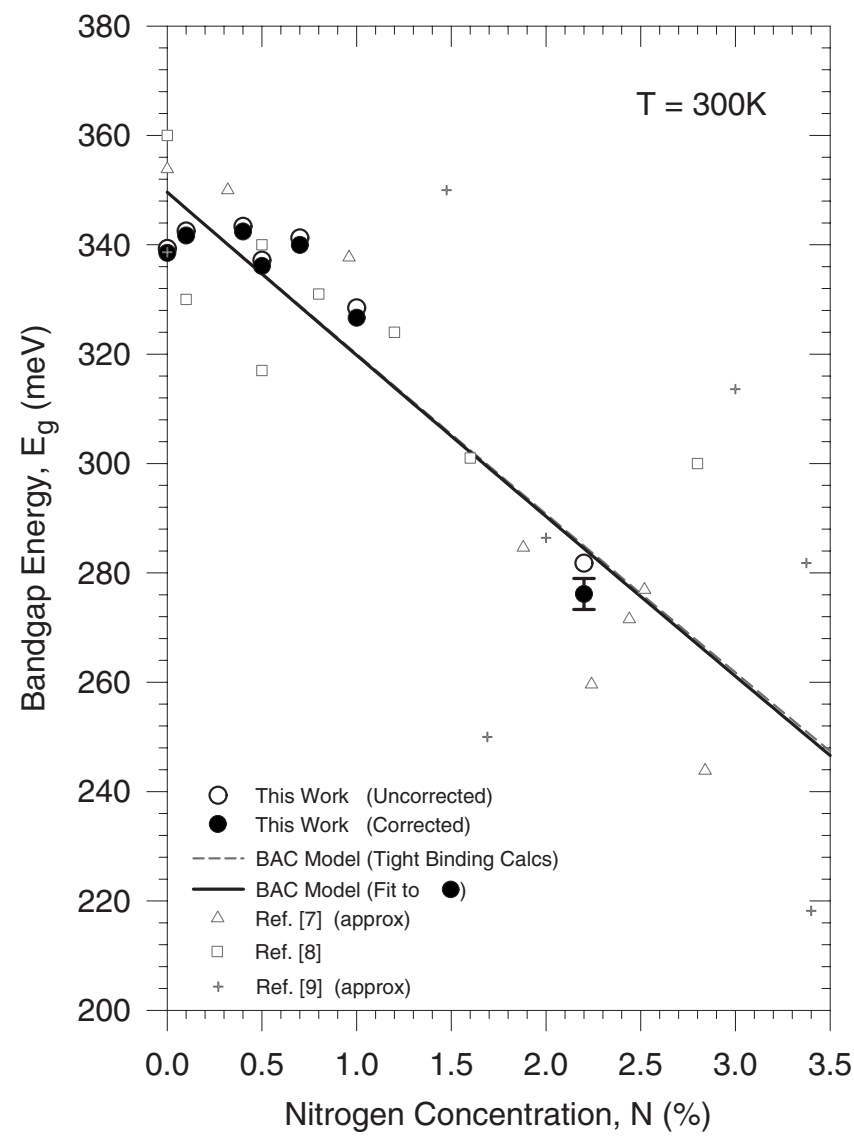

FIG. 8. The filled circles show the experimentally determined $300 \mathrm{~K}$ band-to-band transition energies, obtained by fitting the parabolic line shape model to the measured PL spectra for each $N$ and then applying a correction that accounts for the effects of nonparabolicity (as plotted in Fig. 7.) A representative error bar is shown on the $N=2.2 \%$ data point. Also shown for comparison are the uncorrected transition energies (open circles) as well as the results of absorption measurements published previously by other groups (Refs. 8-10) (smaller symbols; data sets labeled "approx" are estimated from published graphs). The dashed curve shows the theoretical BAC model prediction Eq. (2) based on parameters derived from the tight-binding calculations. The solid curve shows a fit of Eq. (2) to the present experimental results (filled circles), the parameters of which are shown in Table II. There is clearly an excellent correlation between the predicted and fitted BAC model curves (they are virtually coincident), with their gradients indicating a band gap reduction of 29.4 and $29.6 \mathrm{meV} / \%$, respectively.

InNAs for $N \leqslant 2.2 \%$, the values of $E_{g}$ obtained by fitting experimental PL spectra with a parabolic model should simply be corrected downward by an amount $\Delta E_{g}=(0.8$ $\left.+N^{2}\right) \mathrm{meV}$.

By returning now to the parabolic model fits to the experimental $300 \mathrm{~K}$ PL spectra, the open circles in Fig. 8 show the values obtained for $E_{g}$ (para fit) as a function of $N$. The filled circles show the result of applying the above correction for the effects of nonparabolicity [i.e., $E_{g}$ (true) $=E_{g}$ (para fit) $\left.-\left(0.8+N^{2}\right) \mathrm{meV}\right]$. For comparison, we also show some results from absorption measurements published previously by other groups ${ }^{8-10}$ The dashed curve shows the theoretical be- 
TABLE II. Comparison of the theoretical BAC model parameters derived from tight-binding calculations for InNAs at $300 \mathrm{~K}$ (see dashed curve in Fig. 8) and values obtained by fitting Eq. (2) to the experimental values of $E_{g}$ as a function of $N$ (after correction for the nonparabolic conduction-band dispersion, see solid curve in Fig. 8). In this fitting procedure, the initial parameter estimates were taken from the theoretical values shown for $\alpha, \beta, \gamma$, and $E_{N}^{0}$, while for $E_{g}^{\text {InAs }}\left[\equiv E_{M}^{0}(\mathbf{k}=0)\right]$, a value of $350 \mathrm{meV}$ was used, as discussed in the main text. Note that the model is relatively insensitive to changes in $\gamma$, so the uncertainty in its fitted value (not shown) is potentially very large.

\begin{tabular}{lcc}
\hline \hline Parameter & $\begin{array}{c}\text { Theoretical value } \\
(\mathrm{eV})\end{array}$ & $\begin{array}{c}\text { Fit value } \\
(\mathrm{eV})\end{array}$ \\
\hline$\alpha$ & 1.30 & $1.30 \pm 0.52$ \\
$\beta$ & 1.30 & $1.31 \pm 0.19$ \\
$\gamma$ & 2.00 & 2.00 \\
$E_{N}^{0}$ & 1.36 & $1.36 \pm 0.41$ \\
$E_{M}^{0}(\mathbf{k}=0)$ & 0.350 & $0.350 \pm 0.004$ \\
\hline \hline
\end{tabular}

havior predicted by the tight-binding $\mathrm{BAC}$ model parameters (as given in Table II). The full curve shows a fit of the BAC model [i.e., Eq. (2)] to our corrected experimental results (filled circles), using the theoretical BAC model parameters in Table II as initial estimates for fitting the parameters $\alpha, \beta$, $\gamma, E_{N}^{0}$, and $E_{g}^{\text {InAs }}$. As may be concluded from the closeness of the full and dashed curves in Fig. 8, the resulting fitted BAC parameters are in excellent agreement with the original theoretical values, as shown in Table II. These two curves are approximately linear with $N$ over the small concentration range studied and, from their respective gradients, a band gap reduction of $29.6 \mathrm{meV} / \%$ (for $N \leqslant 2.2 \%$ ) is found, which compares well with the gradient of the predicted curve of $29.4 \mathrm{meV} \%$.

Finally, we turn briefly to the PL spectra measured at temperatures below $300 \mathrm{~K}$ (see, for instance, Figs. 2 and 3). Unfortunately, our attempts to fit these spectra with the parabolic model proved less successful than for the $300 \mathrm{~K}$ spectra for all the samples. Although visually satisfactory fits to the experimental spectra could be obtained, the resulting fitted parameters (particularly $E_{e}$ and $E_{h}$ ) were found to be physically unrealistic. We attribute this to the increased contribution of BAC states to the PL emission as the temperature is lowered, which progressively skews the measured PL peaks toward lower energy (see earlier discussion on the S-shaped bowing). The PL line shape fitting function defined by Eqs. (6a) and (6b) is valid for band-to-band recombination only, so the fitting algorithm then attempted to compensate for the change in line shape induced by the BAC states by modifying $E_{e}$ and $E_{h}$. The lower the sample temperature, the greater the contribution from the BAC states, and the more unphysical the fit parameters became. Therefore, a more sophisticated model, capable of modeling multiple recombination paths, is required to analyze the lower temperature PL emission from InNAs.

\section{CONCLUSIONS}

The PL emission from a selection of InNAs epilayers with $N \leqslant 2.2 \%$ has been measured as a means of studying band gap reduction in the alloy system. At both elevated $(300 \mathrm{~K})$ and low temperature $(15 \mathrm{~K})$, an unambiguous and incremental redshift in the emission has been observed as a function of increasing N. A signature S-shaped temperature dependence common to dilute nitride materials is observed in the peak emission energy of several epilayers, the amplitude of which increases with nitrogen content for $N \leqslant 1.0 \%$. However, for $2.2 \% \mathrm{~N}$, the highest concentration studied, little or no S-shaped bowing is apparent. These observations are found to be qualitatively consistent with the predictions of the BAC and LCINS models.

The $300 \mathrm{~K}$ PL spectrum from each epilayer has been fitted with a model that assumes a parabolic conduction-band dispersion in order to obtain band-to-band transition energies $E_{g}$. However, in order to account for the effects of fitting such a parabolic model to the PL spectra of an intrinsically nonparabolic system, theoretical PL spectra have been generated using a fully nonparabolic model for the same material system. Then by least-squares fitting these generated theoretical spectra with the parabolic PL line shape model, a quantitative estimate has been derived for the error in the fitted values of $E_{g}$ due to incorrectly assuming a parabolic conduction-band dispersion. This small and positive error is found to increase as the square of $N$ (for $N \leqslant 2.2 \%$ ). By applying this small error correction to the values for $E_{g}$ obtained from our parabolic fits of the $300 \mathrm{~K}$ experimental PL spectra, more accurate estimates of $E_{g}$ accounting for conduction-band nonparabolicity are, thus, obtained, particularly for $N \geqslant 1.0 \%$. A fit of the BAC model to these corrected $300 \mathrm{~K} E_{g}$ values, plotted as a function of $N$, yields parameters in satisfactory agreement with theoretical values derived from tight-binding calculations. Additionally, a reduction in the $300 \mathrm{~K}$ band gap of $\sim 29.6 \mathrm{meV}$ per $N$ has been estimated from the gradient of this fitted BAC model curve, which is only $0.2 \mathrm{meV}$ per $N$ larger than the corresponding theoretical value.

\section{ACKNOWLEDGMENTS}

The authors thank E. O'Reilly and A. Lindsay for performing tight-binding calculations, from which the theoretical BAC parameters were obtained. The financial support of the UK Engineering and Physical Sciences Research Council (GR/S56030/01) is also acknowledged. 
*j.hosea@surrey.ac.uk

${ }^{1}$ W. Shan, W. Walukiewicz, J. W. Ager III, E. E. Haller, J. F. Geisz, D. J. Friedman, J. M. Olson, and S. R. Kurtz, Phys. Rev. Lett. 82, 1221 (1999).

${ }^{2}$ P. R. C. Kent, L. Bellaiche, and A. Zunger, Semicond. Sci. Technol. 17, 851 (2002).

${ }^{3}$ Dilute Nitride Semiconductors, edited by M. Henini (Elsevier, Amsterdam, 2005).

${ }^{4}$ T. D. Veal, L. F. J. Piper, P. H. Jefferson, I. Mahboob, C. F. McConville, M. Merrick, T. J. C. Hosea, B. N. Murdin, and M. Hopkinson, Appl. Phys. Lett. 87, 182114 (2005).

${ }^{5}$ P. H. Jefferson, T. D. Veal, L. F. J. Piper, B. R. Bennett, C. F. McConville, B. N. Murdin, L. Buckle, G. W. Smith, and T. Ashley, Appl. Phys. Lett. 89, 111921 (2006).

${ }^{6}$ M. Kuroda, R. Katayama, S. Nishio, K. Onabe, and Y. Shiraki, Phys. Status Solidi C 0, 2765 (2003).

${ }^{7}$ S. Kuboya, F. Nakajima, R. Katayama, and K. Onabe, Phys. Status Solidi B 243, 1411 (2006).

${ }^{8}$ M. Kuroda, R. Katayama, K. Onabe, and Y. Shiraki, Phys. Status Solidi B 241, 2791 (2004).

${ }^{9}$ D. K. Shih, H. H. Lin, L. W. Sung, T. Y. Chu, and T. R. Yang, Jpn. J. Appl. Phys., Part 1 42, 375 (2003).

${ }^{10}$ H. Naoi, Y. Naoi, and S. Sakai, Solid-State Electron. 41, 319 (1997)

${ }^{11}$ A. Polimeni, M. Capizzi, M. Geddo, M. Fischer, M. Reinhardt, and A. Forchel, Appl. Phys. Lett. 77, 2870 (2000).
${ }^{12}$ M.-A. Pinault and E. Tournié, Appl. Phys. Lett. 78, 1562 (2001).

${ }^{13}$ A. Lindsay and E. P. O’Reilly, Phys. Rev. Lett. 93, 196402 (2004).

${ }^{14}$ Y. P. Varshni, Physica (Amsterdam) 34, 149 (1967).

${ }^{15}$ H. B. Bebb and E. W. Williams, Semicond. Semimetals 8, 238 (1972).

${ }^{16}$ Y. Lacroix, C. A. Tran, S. P. Watkins, and M. L. W. Thewalt, J. Appl. Phys. 80, 6416 (1996).

${ }^{17}$ J. Plaza, J. L. Castano, B. J. Garcia, H. Carrere, and E. BedelPereira, Appl. Phys. Lett. 86, 121918 (2005).

${ }^{18}$ X. Gong, H. Kan, T. Yamaguchi, I. Suzuki, M. Aoyama, M. Kumagawa, N. L. Rowell, A. Wang, and R. Rinfret, Jpn. J. Appl. Phys., Part 1 33, 1740 (1994).

${ }^{19}$ Z. M. Fang, K. Y. Ma, D. H. Jaw, R. M. Cohen, and G. B. Stringfellow, J. Appl. Phys. 67, 7034 (1990).

${ }^{20}$ H. P. D. Schenk, M. Leroux, and P. de Mierry, J. Appl. Phys. 88, 1525 (2000).

${ }^{21}$ H. Yaguchi, S. Kikuchi, Y. Hijikata, S. Yoshida, D. Aoki, and K. Onabe, Phys. Status Solidi B 228, 273 (2001).

${ }^{22}$ C. R. Pidgeon and R. N. Brown, Phys. Rev. 146, 575 (1966).

${ }^{23}$ I. Vurgaftman, J. R. Meyer, and L. R. Ram-Mohan, J. Appl. Phys. 89, 5815 (2001).

${ }^{24}$ B. Ullrich and C. Bouchenaki, Jpn. J. Appl. Phys., Part 1 30, 1285 (1991).

${ }^{25}$ M. Fisher and A. Krier, Infrared Phys. Technol. 38, 405 (1997). 